\title{
Comparison between DXA and MRI for the Visceral Fat Assessment in Athletes
}

(๑) $(\Theta \Theta$

\author{
Authors \\ Hiroko Murata1, 2, Tomoyoshi Yagi³, Taishi Midorikawa4, Suguru Torii ${ }^{1,5}$, Eri Takai ${ }^{1,6}$, Motoko Taguchi', 5
}

Affiliations

1 Waseda Institute of Sports Nutrition, Waseda University, Tokorozawa, Japan

2 Department of Health and Nutrition, Jumonji University, Saitama, Japan

3 Graduate school of Sport Sciences, Waseda University, Saitama, Japan

4 College of Health and Welfare, J F Oberlin University, Tokyo, Japan

5 Faculty of Sport Sciences, Waseda University, Saitama, Japan

6 Sports Medical Center, Japan Institute of Sports Sciences, Tokyo, Japan

Key words body composition, metabolic syndrome, heavyweight, abdominal obesity, obese

accepted 17.11.2021

published online $\quad 02.02 .2022$

Bibliography

Int J Sports Med 2022; 43: 625-631

DOI 10.1055/a-1717-1619

ISSN $\quad 0172-4622$

(C) 2022. The Author(s).

This is an open access article published by Thieme under the terms of the Creative Commons Attribution-NonDerivative-NonCommercial-License, permitting copying and reproduction so long as the original work is given appropriate credit. Contents may not be used for commecial purposes, or adapted, remixed, transformed or built upon. (https://creativecommons. org/licenses/by-nc-nd/4.0/)

Georg Thieme Verlag KG, Rüdigerstraße 14,

70469 Stuttgart, Germany

\section{Correspondence}

Dr. Motoko Taguchi

Waseda University

Faculty of Sport Sciences

2-579-15 Mikajima

3591192 Tokorozawa

Japan

Tel.: + 81/4/2947 6778, Fax: + 81/4/29476778

mtaguchi@waseda.jp

\section{ABSTRACT}

The use of dual-energy X-ray absorptiometry (DXA) may be invalid for assessing athletes with larger bodies, larger lean body mass, and thicker trunks. This study compared the athletes' visceral adipose tissue (VAT) assessed using DXA and magnetic resonance imaging (MRI). Eighty-two Japanese male collegiate athletes from 18 sports participated in this study. VAT was assessed using the dual-energy scan that coincided with the 4th lumbar vertebra. The sum of eight magnetic resonance slices corresponding to the region of the dual-energy scan was used for comparison. The VAT volume was higher with the dual-energy scan than with MRI (difference: $35 \mathrm{~cm}^{3}, \mathrm{p}<0.01$ ). A significant correlation was noted between the volumes measured using both modalities $(r=0.88, p<0.01)$. Magnetic resonance-derived volumes less than $600 \mathrm{~cm}^{3}$ showed a stronger significant correlation with dual-energy-derived volumes. However, magnetic resonance-derived VAT volumes exceeding $600 \mathrm{~cm}^{3}$ were not significantly correlated with dual-energy-derived volumes. In conclusion, VAT volumes derived from DXA were larger and significantly correlated with those derived from MRI across a wide range of values. Methods using DXA for assessing the visceral fat volume may require adjustment to estimate abdominal visceral fat volume in athletes, with care taken when using such methods for heavyweight athletes.

\section{Introduction}

Visceral fat accumulation is a strong, independent predictor of allcause mortality, dyslipidemia, and insulin resistance in men in the general population [1-4]. Recent studies have reported that the excessive accumulation of body fat and visceral fat is associated with cardiometabolic risk in athletes. Some studies have shown that linemen have a high body fat ratio and insulin resistance [5, 6]. It has also been reported that heavyweight athletes have more visceral fat and a higher cardiometabolic risk than non-heavyweight athletes in Judo and American football [7]. Considering these cases, excessive visceral fat accumulation should not be ignored. It could contribute to poor health in athletes, such as metabolic syndrome, 
insulin resistance, dyslipidemia, and increased body fat percentage. However, evidence regarding the measurement methods for visceral fat accumulation in athletes is inadequate. The "gold standard" for measuring and analyzing visceral fat comprises magnetic resonance imaging (MRI) and computed tomography (CT). These methods are highly accurate [8-11]; however, they require many resources, such as an expensive device and software for analysis, and CT scans are inappropriate for athletes because they cause radiation exposure. MRI is occasionally used to assess muscle volume in athletes [12]; however, it is costly and labor-intensive.

Recently, dual-energy X-ray absorptiometry (DXA) has been used to estimate visceral fat volume. These methods can estimate the visceral adipose tissue (VAT). The MRI method evaluated the VAT by analysis of the abdominal slice images, and the DXA method assessed by automatic analysis of the partial image of the interest area. These methods measure the adipose tissue within the inner abdominal muscle wall $[13,14]$. Volume of VAT assessed by MRI (MRI-VAT) was determined by calculating the VAT volume from a single abdominal cross-sectional image. The surgeons used their cursor to identify VAT and subcutaneous adipose tissue (SAT) areas on a computer monitor during MRI analysis. They set one color for the adipose tissue area in the intestines, bones, and muscles within the inner abdominal muscle wall for VAT and set another color on adipose tissue outside the abdominal muscle wall for SAT in each abdominal cross-sectional image. The reproducibility of these methods during MRI analysis was determined by the surgeon's skill. Volume of VAT assessed by DXA (DXA-VAT) was measured in a $5-\mathrm{cm}$ wide region placed across the entire abdomen just above the iliac crest at a level that nearly coincided with the $4^{\text {th }}$ lumbar vertebrae on the whole body DXA scan.

The DXA measures both the visceral fat and the subcutaneous fat. However, on each side of the abdominal cavity, the DXA scan directly measured the subcutaneous fat. The lateral abdominal subcutaneous fat seen in the interest DXA image was used to estimate the anterior and posterior subcutaneous abdominal fat, allowing the VAT to be estimated from the total abdominal fat measured $[15,16]$. However, this automated analysis by DXA measurements may increase VAT estimation. The use of DXA for visceral fat assessment is faster and less labor-intensive. Some previous studies have reported that DXA provides a valid assessment of visceral fat for the general population $[15,16]$. However, a previous pilot study reported that DXA may provide reasonable descriptive cross-sectional body composition data for obese cohorts but is less robust in providing accurate individual values [17]. Only one study has reported the difference in visceral fat volume between positions in athletes belonging to the National Football League using DXA assessment [18]. However, DXA assessment for visceral fat in athletes may be invalid because such athletes have larger bodies, a larger lean body mass, and thicker trunks. Thus, a comparison between DXA and conventional methods, such as MRI, seems necessary to assess visceral fat. This is especially true for MRI, which is commonly used to assess muscle volume in athletes.

Therefore, this study aimed to compare DXA and MRI regarding visceral fat volume assessment in athletes. We hypothesize that DXA measurements are similar to the MRI volume measurements in most cases; however, the margin on similarity in large athletes widens considerably.

\section{Materials and Methods}

\section{Subjects}

A total of 82 Japanese male collegiate athletes were recruited from the following sports: volleyball $(n=12)$, judo $(n=9)$, sumo $(n=8)$, ski $(n=7)$, water polo $(n=7)$, body building $(n=7)$, American football $(n=6)$, baseball $(n=6)$, weightlifting $(n=6)$, cycling $(n=4)$, martial arts $(n=3)$, swimming $(n=2)$, running $(n=1)$, soccer $(n=1)$, basketball $(n=1)$, lacrosse $(n=1)$, and tennis $(n=1)$. Male collegiate athletes with regular training at high intensity were recruited to participate in this study. Exclusion criteria included candidates currently taking medication, in poor health or with metal implants that prevented them from successfully undergoing DXA and MRI scans. All participants were adequately informed about the study both verbally and in writing. Each participant provided written informed consent before starting the study. This study was conducted ethically following the standards described by the International Journal of Sports Medicine [19] and approved by our institution's ethics committee regarding the use of human subjects, in accordance with the Declaration of Helsinki.

\section{Measurements}

The height and body weight of the subjects were measured. Next, DXA and MRI scans were performed randomly. All measurements were completed within approximately 20 minutes for the same biological condition of measurements in each subject. The participants' heights were measured to the nearest $0.1 \mathrm{~cm}$ using a stadiometer, and the body weight was measured to the nearest $0.05 \mathrm{~kg}$ using a digital scale. During this time, the subjects wore light clothing and were barefoot. Waist circumference was measured using a flexible steel tape measure (Rosscraft Innovations, Canada) at the level of the narrowest line between the lower costal (10th rib) border and the top of the iliac crest perpendicular to the long axis of the trunk. This anthropometric measurement was measured by a Level 1 ISAK practitioner, which needed $\leq 1.5 \%$ of technical error of measurements (TEM) for all the anthropometric measurements except for the skinfold thickness within the intra practitioner. The TEM of the waist circumference by this practitioner was $0.2 \%$.

Whole-body DXA scans were performed using a Hologic DXA machine (Horizon A configured with APEX version 5.6, Hologic Inc., MA, USA). The DXA field of view was $195 \times 65 \mathrm{~cm}$, and the DXA table weight limit was $204 \mathrm{~kg}$. The DXA measurements were carried out by a skillful orthopedic surgeon and radiographer, who were coauthors in this study. The precision of the Hologic Horizon A, the same model we used in our study, has reported the CV\% of the total body fat and total body lean mass were less than $1 \%$ [20,21]. MRI slices were obtained using Signa 1.5 T (General Electric Co. Ltd., WI, USA). The imaging conditions included a T1-weighted spin-echo and axial-plane sequence with a slice thickness of $6.5 \mathrm{~mm}$, a repetition time of $400 \mathrm{~ms}$, and an echo time of $7.8 \mathrm{~ms}$. The images were transferred to a personal computer in the DICOM file format. Crosssectional areas of VAT were determined using digital image analysis software (Slice-O-Matic 4.3, for Windows; Tomovision, Canada). The coefficient of variance was $3.8 \%$, and the technical error of the measurement was $4.4 \%$ for VAT. 


\section{Analysis for comparison}

A previous validation study assessing visceral fat volume using a DXA device reported that DXA-VAT volume was measured in a 5-cm-wide region across the entire abdomen just above the iliac crest at a level that approximately coincided with the 4th lumbar vertebra on whole-body DXA [15]. The width of each scan in the DXA machine was $13 \mathrm{~mm}$ [14]. From this information, a 5-cm-wide region across the entire abdomen, measured by the DXA machine, coincided with approximately four times its scan area. Therefore, the thickness of the MRI slices to compare DXA and MRI volume was determined to be $6.5 \mathrm{~mm}$, which was half of the 13-mm-wide DXA scan, to allow for more precise measurements. Eight MRI slices were used to match the DXA region for visceral fat assessment ( $\vee$ Fig. 1). Abdominal thickness was determined as the highest anterior-posterior abdominal height and measured by using a ruler in digital image analysis software in eight MRI slice images.

\section{Statistical analysis}

The sample size of the study was calculated with the $G$ * power 3 software, with a power of 0.8 , a two-tailed significance level of 0.05 , and an effect size of 0.3 [22]. Data are shown as mean \pm standard deviation for normally distributed data and median (interquartile range) for non-normally distributed data. The KolmogorovSmirnov test was performed to assess the normality of data distribution, and non-normally distributed data were log-transformed before analysis. DXA-VAT and MRI-VAT volumes were compared. The difference and total error were calculated as follows:

Difference $=$ DXA-VAT - MRI-VAT

Total error $=($ DXA-VAT - MRI-VAT $) 2^{\wedge} 0.5$

The Mann-Whitney test was used to assess significant differences between the DXA-VAT and MRI-VAT volumes. Moreover, we executed a single regression analysis to examine the relationship between MRI-VAT and DXA-VAT. Linear regression was used to compare both volumes, and Pearson's correlation coefficients were computed to compare both volumes. Bland-Altman analysis was used to assess the magnitude of bias and systematic errors [23]. Data were analyzed using IBM SPSS ver. 24 (IBM Japan Inc., Tokyo, Japan). The significance level was set at $\mathrm{p}<0.05$.

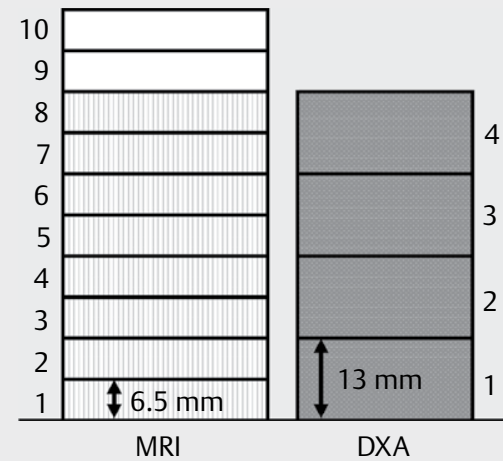

The superior edge of the illiac crest

- Fig. 1 MRI slices that coincided with the estimated DXA-VAT region. One DXA slice was equal to two MRI slices. DXA, dual-energy absorptiometry; MRI, magnetic resonance imaging; VAT, visceral adipose tissue.

\section{Results}

The anthropometric data and body composition characteristics of the subjects are shown in $>$ Table 1 . A histogram of the volume measured with usual MRI methods is presented in > Fig. 2 . Most of the subjects belonged to groups with MRI-VAT volumes ranging from 100 to $300 \mathrm{~cm}^{3}$. However, some subjects were included in a wider range of $>400 \mathrm{~cm}^{3}$ to $<800 \mathrm{~cm}^{3}$. The results of statistical comparisons between MRI-VAT and DXA-VAT are presented in $>$ Table 2. Our findings showed that the DXA-VAT volume was $35(-1-63) \mathrm{cm}^{3}$ larger than the MRI-VAT volume, and the difference was significant $(p<0.01)$. Additionally, the total error, which explains the magnitude of the difference between the DXA-VAT and MRI-VAT volumes, was $43(15-78) \mathrm{cm}^{3}$. This total error value, which was $43 \mathrm{~cm}^{3}$, exceeded the technical measurements error for the reference MRI method by $4.4 \%$ (about $9.5 \mathrm{~cm}^{3}$ ) for comparison. The relationship between MRIVAT and DXA-VAT is shown in > Fig. 3. A significant correlation was observed between DXA-VAT volume and MRI-VAT volume $(r=0.88$, $p<0.01)$. The line of fit for the relationship between DXA and MRI-

- Table 1 Anthropometric and body composition characteristics of the subjects $(n=82)$.

\begin{tabular}{|c|c|}
\hline Variable & \\
\hline Age (years) & $20(19-21)$ \\
\hline Height $(\mathrm{cm})$ & $175.3 \pm 7.2$ \\
\hline Body weight (kg) & $75.0(69.9-87.6)$ \\
\hline BMI $\left(\mathbf{k g} / \mathrm{m}^{2}\right)$ & $24.3(22.8-28.3)$ \\
\hline DXA BF (\%) & $12.9(9.9-17.1)$ \\
\hline DXA FM (kg) & $9.4(6.9-14.7)$ \\
\hline DXA FFM (kg) & $66.0(60.2-74.9)$ \\
\hline Waist circumference $(\mathrm{cm})$ & $78.3(75.0-87.5)$ \\
\hline MRI-VAT volume $\left(\mathrm{cm}^{3}\right)^{\dagger}$ & $217(184-287)$ \\
\hline DXA-VAT volume $\left(\mathrm{cm}^{3}\right)$ & $250(215-319)$ \\
\hline Abdominal thickness $(\mathrm{cm})^{\ddagger}$ & $18.0(17.1-19.8)$ \\
\hline
\end{tabular}

Data are shown as mean \pm SD for normally distributed data and as median (interquartile range) for non-normally distributed data; ${ }^{\dagger} \mathrm{MRI}$ volume was measured in a $5.2-\mathrm{cm}$-wide region across the entire abdomen just above the iliac crest. ¥Abdominal thickness: Highest anterior-posterior abdominal height in eight cross-sectional MRI scans; BF, body fat; FM, fat mass; FFM, fat free mass; BMI, body mass index; DXA, dual-energy absorptiometry; MRI, magnetic resonance imaging; VAT, visceral adipose tissue

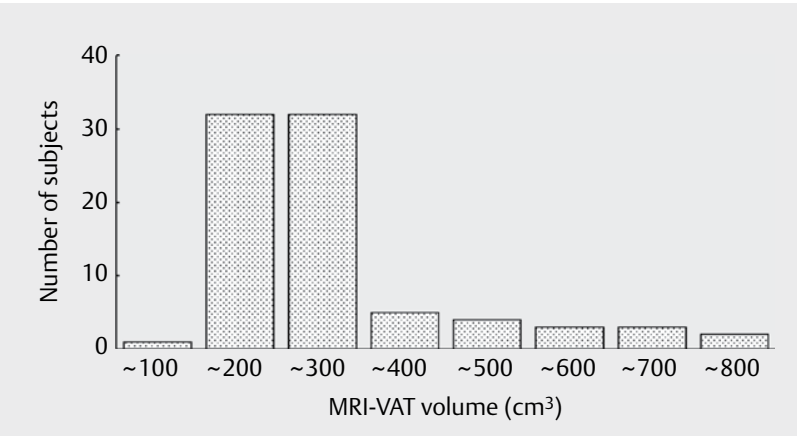

- Fig. 2 Histogram of VAT volume measured by MRI $(n=82)$. MRI, magnetic resonance imaging; VAT, visceral adipose tissue. 
- Table 2 Comparison of MRI- and DXA-VAT volumes $(n=82)$.

\begin{tabular}{|l|l|l|l|l|}
\hline & VAT volume $\left.\mathbf{( c m}^{\mathbf{3}}\right)$ & Difference $^{*}$ & Total error $^{\dagger}$ & $\mathbf{p}^{\ddagger}$ \\
\hline MRI & $217(184-287)$ & & & \\
\hline DXA & $250(215-319)$ & $35(-1-63)$ & $43(15-78)$ & $\mathrm{p}<0.01$ \\
\hline
\end{tabular}

Data are shown as median (interquartile range); ${ }^{*}$ Corresponds to DXA minus MRI volume; ${ }^{\dagger}$ Corresponds to the square root of the square of DXA-VAT volume minus MRI-VAT volume; $\ddagger \mathrm{p}$-value from Mann-Whitney test for a significant difference between DXA-VAT and MRI-VAT; MRI, magnetic resonance imaging; DXA, dual energy X-ray absorptiometry; VAT, visceral adipose tissue.

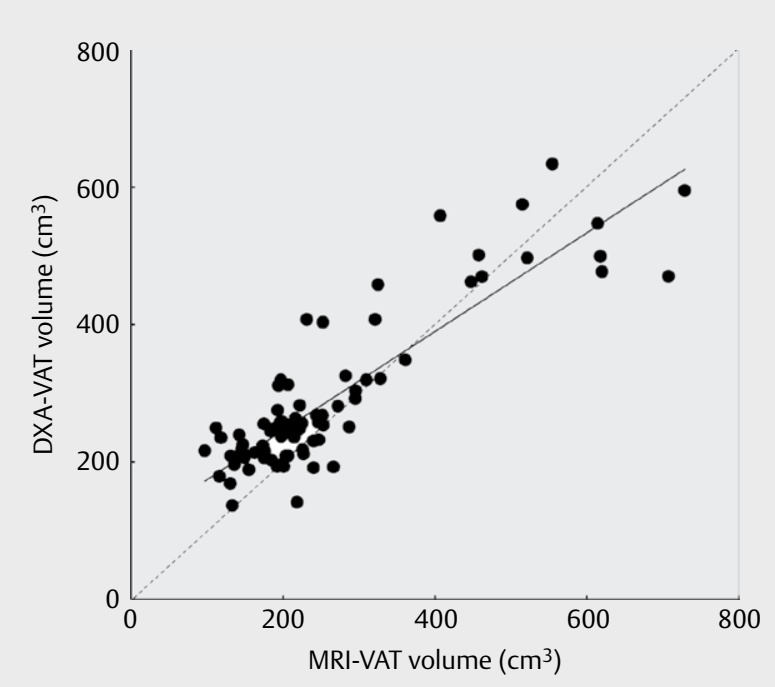

- Fig. 3 Relationship between DXA-VAT volume and MRI-VAT volume. DXA, dual-energy absorptiometry; MRI, magnetic resonance imaging; VAT, visceral adipose tissue.

VAT was calculated as follows: DXA-VAT volume $=0.72 \times$ MRI-VAT volume $+102.36\left(\mathrm{~cm}^{3}\right)$. Although all the data in $\mathbf{F i g . ~} \mathbf{3}$ showed significant correlation, the slope of this regression line was 0.72 less than 1 , and its intercept was 102.36 more than 0 . Therefore, the congruence between the methods was not established. Especially, there was some lack of congruence between the measurements at high VAT volumes ( $>$ Fig. 3). Bland-Altman analysis was conducted to examine systematic errors. The Bland-Altman bias was $+29 \mathrm{~cm}^{3}$ for the MRI-VAT volume according to the usual methods. The $95 \%$ limit of agreement was -105 to $+163 \mathrm{~cm}^{3}$. A significant negative correlation was observed between the difference and the average of the DXA-VAT and MRI-VAT volumes $(r=-0.381, p<0.001)$ ( $>$ Fig. 4). Five subjects had MRI-VAT volumes greater than $600 \mathrm{~cm}^{3}$. The median DXA-VAT volume in the five subjects whose MRI-VAT volume exceeded $600 \mathrm{~cm}^{3}$ was $499 \mathrm{~cm}^{3}$ (range $470-595 \mathrm{~cm}^{3}$ ). The median body mass was $114.0 \mathrm{~kg}$ (range 107.5-123.6 kg), the median total body fat was $26.2 \mathrm{~kg}$ (range $23.5-31.9 \mathrm{~kg}$ ), and the median abdominal thickness was $25.0 \mathrm{~cm}$ (range $24.1-26.3 \mathrm{~cm}$ ). To examine a congruence with the line of identity in some range of the MRI-VAT volume, Bland-Altman plot analysis on MRI-VAT data set of below $700 \mathrm{~cm}^{3}$ and $600 \mathrm{~cm}^{3}$ were examined. Systematic error shown as significant tendency of correlation was slightly detected below $700 \mathrm{~cm}^{3}$ of the MRI-VAT volume ( Fig. 5, $r=-0.204, p=0.069$ ); however, it was not

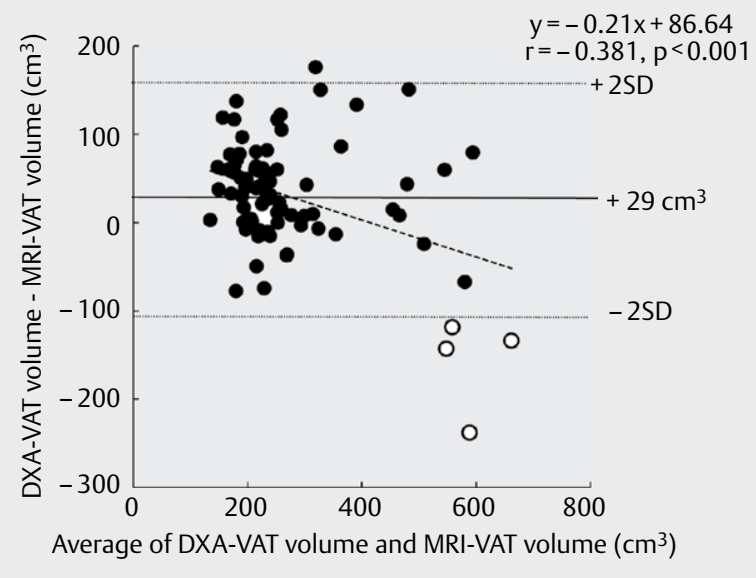

- Fig. 4 Bland-Altman plot of DXA-VAT volume against MRI-VAT volume. The solid line marked at $35 \mathrm{~cm}^{3}$ represents the difference between the DXA and MRI volumes. The non-solid circles represent four subjects who were outside the $95 \%$ lower limit of agreement in accordance with the Bland-Altman analysis. DXA, dual-energy absorptiometry; MRI, magnetic resonance imaging; VAT, visceral adipose tissue.

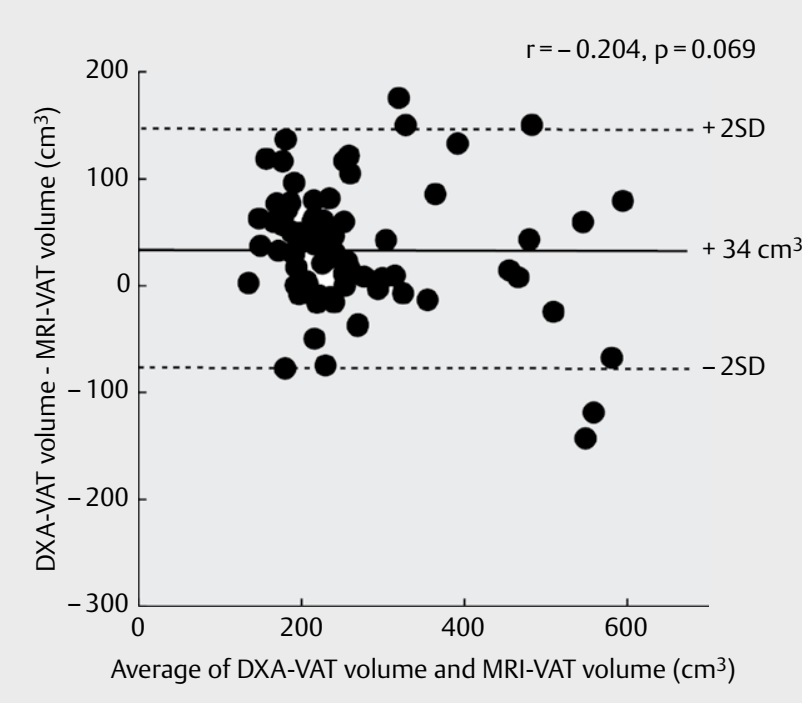

- Fig. 5 Relationship between DXA-VAT volume and MRI-VAT volume in limited data below $700 \mathrm{~cm}^{3}$ in MRI-VAT volume. DXA, dualenergy absorptiometry; MRI, magnetic resonance imaging; VAT, visceral adipose tissue.

detected below $600 \mathrm{~cm}^{3}$ ( $\vee$ Fig. 6, $r=0.078, p=0.500$ ). We further examined the relationship between the MRI-VAT and DXA-VAT volumes by dividing MRI-VAT into two ranges based on a cutoff of $600 \mathrm{~cm}^{3}$ for MRI-VAT volume ( $\mathbf{F i g}$. 7). A significant correlation was observed between the DXA-VAT and MRI-VAT volumes for MRI-VAT volumes below $600 \mathrm{~cm}^{3}$, and the correlation coefficient for the regression was 0.89 . The line of fit for the relationship was calculated as follows: DXA-VAT volume $=0.90 \times$ MRI-VAT volume $+63\left(\mathrm{~cm}^{3}\right)$. 


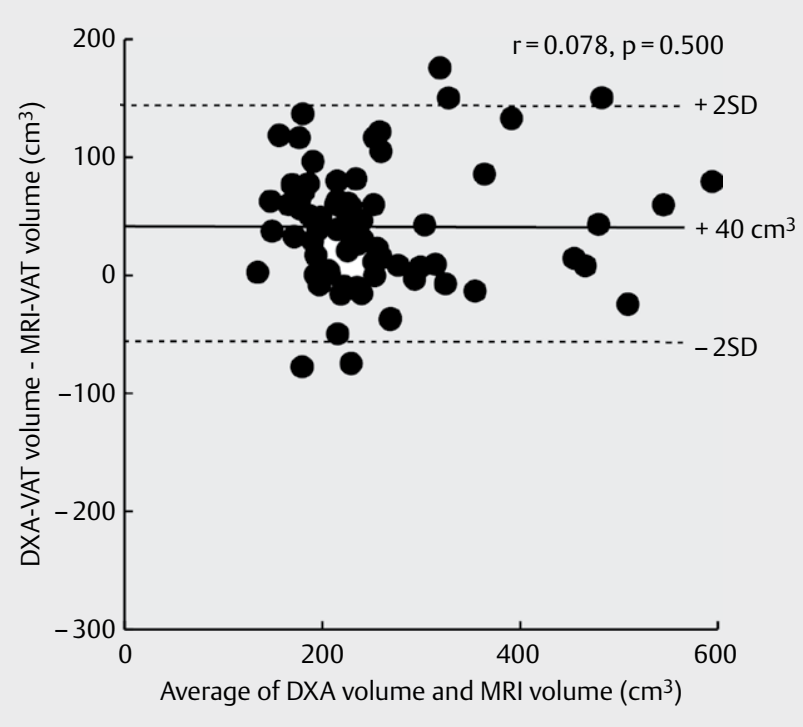

- Fig. 6 Relationship between DXA-VAT volume and MRI-VAT volume in limited data below $600 \mathrm{~cm}^{3}$ in MRI-VAT volume. DXA, dualenergy absorptiometry; MRI, magnetic resonance imaging; VAT, visceral adipose tissue.

However, a significant correlation was not observed between DXAVAT and MRI-VAT volumes for MRI-VAT volumes above $600 \mathrm{~cm}^{3}$.

\section{Discussion}

In this study, we compared DXA and MRI assessments of visceral fat volume in athletes. The main result of our study was that a significant correlation was shown between the DXA-VAT and MRI-VAT volumes $(r=0.88, p<0.01)$. However, this relationship was not significant for subjects whose MRI-VAT volumes were large. Therefore, the additional examination of validity for DXA -VAT method using Bland-Altman analysis was carried out ( $>$ Figs. 5-7). These results found that DXA assessment has poor validity for subjects with MRIVAT greater than $600 \mathrm{~cm}^{3}$.

Micklesfield et al. reported that the assessment by DXA and a clinical read of VAT from a CT scan in their previous study of the general population in South Africa. Additionally, they showed a strong correlation between DXA assessment and $C T$, indicating that DXA assessment modality was similar to CT $(r=0.93)$ [15]. Another validation study that assessed VAT in the Indian population using DXA versus MRI as the usual method showed that DXA measurements for abdominal fat were suitable for use [24]. A study reported that VAT measurement by DXA displayed a satisfactory level of precision in individuals with a body mass index (BMI) of $>18.5 \mathrm{~kg} /$ $\mathrm{m}^{2}$ [25]. However, previous studies of obese people showed that DXA assessments for VAT were not reliable enough. A high body fat percentage and BMI especially affected the precision error as noted in our results. One study reported that the precision error showed as root-mean square error standard deviation and percent coefficient of variation for VAT mass measured twice by DXA in an obesity group was smaller than that in the overweight and normal-

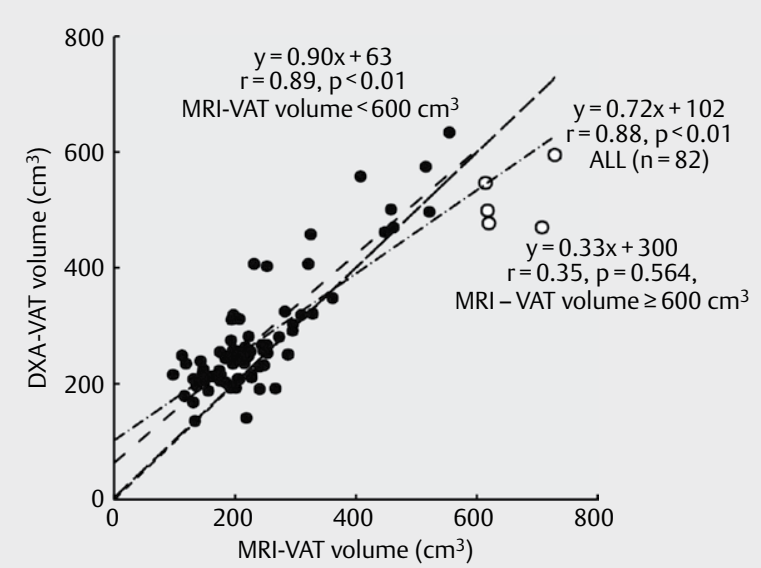

- Fig. 7 Relationship between DXA-VAT volume and MRI-VAT volume. The solid circles indicate values below $600 \mathrm{~cm}^{3}$ in MRI-VAT volume and the non-solid circles indicate volumes greater or equal to $600 \mathrm{~cm}^{3}$. DXA, dual-energy absorptiometry; MRI, magnetic resonance imaging; VAT, visceral adipose tissue.

weight groups. However, caution should be taken in estimating obese adults with a large VAT mass [26]. Another study showed that an increased BMI and body fat percentage had a clinically significant effect on precision errors, with larger precision errors in those with higher BMI and body fat. This effect was mostly marked for the sub-regional measurements with the lowest precision errors in the body [27]. Moreover, LaForgia et al. reported that although DXA provided average body fat percentage data for obese adults without bias compared to data obtained using a four-compartment body composition model, individual data were associated with large prediction errors. They also showed that body fat percentage assessed by DXA was underestimated compared with that obtained using a four-compartment body composition model in a large body [17]. The methodology review article that used DXA to assess body composition in athletes has shown the scan mode or speed for accuracy. A typical DXA machine generally has three scan modes that adjust the $X$-ray attenuation for the thickness of each subject: thin $(<13 \mathrm{~cm})$, standard $(13-25 \mathrm{~cm})$, and thin $(>25 \mathrm{~cm})$, although initially automatically determined by the software from the subject's body mass index, this can be overridden by the technician [28] and the operator's manual of DXA device used in this study also recommended measurements using the automatic mode (14).

However, this automatic VAT determination by DXA measurements may increase VAT estimation. Because the supine position causes a shift of abdominal adipose tissue with SAT extending laterally, VAT differentiated from total abdominal fat of interest from the abdominal image by DXA may be estimated increasingly.

Five subjects whose results were also included above were assessed by MRI to have VAT volumes exceeding $600 \mathrm{~cm}^{3}$. They had a larger body mass, total body fat than the other subjects. High body fat percentage, excess body fat in heavyweight athletes may lead to precision errors in measuring fatness parameters, as seen 
in previous studies of obese populations. Therefore, the assessment of VAT by DXA for heavyweight athletes with very high MRI-VAT volumes is difficult and may result in an underestimation of their VAT compared with MRI-measured VAT. Additionally, VAT assessed by MRI for those with a much larger body mass was not significantly correlated with DXA-VAT.

There were some limitations to this study. First, the subjects comprised only male collegiate athletes. Previous studies have reported that high school athletes also have a risk of metabolic syndrome [29]. Watanabe et al. reported that the risk of metabolic syndrome increased with the degree of fatty liver for judo athletes with pre-metabolic syndrome in elementary school's fourth to sixth grades [30]. Hurst et al. reported that retired linemen had higher cardiometabolic risk [31]. Future studies are needed to collect data on a wider age range, such as teenage to middle-aged athletes. Second, only male athletes were chosen as participants for this study. The risk of metabolic syndrome related to visceral fat accumulation is higher in male athletes than in female athletes. Few studies in female athletes have reported the risk of metabolic syndrome and visceral fat accumulation. However, some female athletes of sports, such as judo and weightlifting, have heavy body mass. In the future, the study of visceral fat assessment and the resulting metabolic syndrome in female athletes is warranted. Third, this study had inadequate data on heavyweight athletes with a very high body mass and visceral fat; therefore, a significant correlation for large VAT values might not have been noted. Further, we examined the correlation between DXA-VAT and MRI-VAT using a cutoff of $600 \mathrm{~cm}^{3}$. However, we could not determine whether this cutoff was suitable. Although the sample size was determined using $G$ power, comparative studies of athletes with a wider range of body sizes may require larger sample sizes. Additional research is needed to confirm and extend this finding. Lastly, a 1.5 Tesla MRI was used in this study; however, a 3 Tesla MRI was used in previous studies. Using the lower field strength MRI instrument compared with a 3 Tesla MRI may influence the measurements. A higher field strength MRI than used in this study should be considered in further studies. However, the previous study that used a 3 Tesla MRI machine reported that VAT measured by DXA was underestimated compared to that measured using a higher field strength MRI [32]. The results of this previous study were different from that of our study. Although the subjects in our study were young male athletes, the subjects in this previous study were adolescents. Moreover, the difference between the MRI devices used in our study and those used in this previous study was field strength and this might influence the results of studies involving VAT volume. Therefore, further studies for athletes using high field strength might be required. Additionally, another recent previous study showed that DXA and MRI VAT are interchangeable when measured over an anatomically matched abdomen region, while subcutaneous adipose tissue (SAT) and VAT/SAT ratio differ between the two modalities. However, the MRI-VAT of the two participants groups being measured by different field strength MRI was considered a problem [33]. These problems may occur in contemporary cohort studies. Moreover, another previous study about the precision of the Hologic DXA in the assessment of VAT reported that VAT measurement by Hologic DXA displays a satisfactory level of precision on individuals with a BMI of $>18.5 \mathrm{~kg} / \mathrm{m}^{2}$ [25]. However, our study showed that
VAT measurements for subjects with larger VAT measured by MRI presented poor validity. Further studies of VAT assessment by DXA for heavyweight athletes with large body are required.

In conclusion, VAT volumes derived from DXA were larger and significantly correlated with those derived from MRI across a wide range of values. Therefore, methods using DXA for assessing visceral fat volume may require adjustment to estimate abdominal visceral fat volume in athletes, with care taken when using such methods for heavyweight athletes. Future studies should examine other obesity-related parameters, such as body weight, body fat percentage, and abdominal thickness, and not only the relationship between DXA-VAT and MRI-VAT, in athletes whose visceral fat volume is assessed by DXA is suitable. Additionally, common or regular use of methods, such as DXA, that are acceptable to athletes for assessing visceral fat volume might improve athlete conditioning and health by preventing many diseases caused by excess body fat, especially visceral abdominal fat.

\section{Conflict of Interest}

The authors declare that they have no conflict of interest.

\section{References}

[1] Kuk JL, Katzmarzyk PT, Nichaman MZ et al. Visceral fat is an independent predictor of all-cause mortality in men. Obesity (Silver Spring) 2006; 14: 336-341

[2] Despres JP, Moorjani S, Lupien PJ et al. Regional distribution of body fat, plasma lipoproteins, and cardiovascular disease. Arteriosclerosis 1990; 10: 497-511

[3] Banerji MA, Buckley MC, Chaiken RL et al. Liver fat, serum triglycerides and visceral adipose tissue in insulin-sensitive and insulin-resistant black men with NIDDM. Int J Obes Relat Metab Disord 1995; 19: 846-850

[4] Neeland IJ, Ross R, Després JP et al. Visceral and ectopic fat atherosclerosis, and cardiometabolic disease: a position statement. Lancet Diabetes Endocrinol 2019; 7: 715-725

[5] Borchers JR, Clem KL, Habash DL et al. Metabolic syndrome and insulin resistance in Division 1 collegiate football players. Med Sci Sports Exerc 2009; 41: 2105-2110

[6] Buell JL, Calland D, Hanks $F$ et al. Presence of metabolic syndrome in football linemen. J Athl Train 2008; 43: 608-616

[7] Murata H, Oshima S, Torii S et al. Characteristics of body composition and cardiometabolic risk of Japanese male heavyweight Judo athletes. J Anthropol Physiol 2016; 35: 10: 1-6

[8] Klopfenstein B], Kim MS, Krisky CM et al. Comparison of 3 T MRI and $\mathrm{CT}$ for the measurement of visceral and subcutaneous adipose tissue in humans. Br J Radiol 2012; 85: e826-e830

[9] Lee YH, Hsiao HF, Yang HT et al. Reproducibility and repeatability of computer tomography-based measurement of abdominal subcutaneous and visceral adipose tissues. Sci Rep 2017; 7: 40389

[10] Abate N, Burns D, Peshock RM et al. Estimation of adipose tissue mass by magnetic resonance imaging: validation against dissection in human cadavers. J Lipid Res 1994; 8: 1490-1496

[11] Rössner S, Bo W], Hiltbrandt E et al. Adipose tissue determinations in cadavers- a comparison between cross-sectional planimetry and computed tomography. Int J Obes 1990; 10: 893-902 
[12] Midorikawa T, Sekiguchi O, Beekley MD et al. A comparison of organ-tissue level body composition between college-age male athletes and nonathletes. Int J Sports Med 2007; 28: 100-105

[13] Shuster A, Patlas M, Pinthus ] et al. The clinical importance of visceral adiposity: a critical review of methods for visceral adipose tissue analysis. Br J Radiol 2012; 85: 1-10

[14] Hologic Inc Horizon QDR Series operator manual Document No. MAN-03644 Revision 0032015

[15] Micklesfield LK, Goedecke JH, Punyanitya M et al. Dual-energy X-ray performs as well as clinical computed tomography for the measurement of visceral fat. Obesity (Silver Spring) 2012; 20: 1109-1114

[16] Kaul S, Rothney MP, Peters DM et al. Dual-energy X-ray absorptiometry for quantification of visceral fat. Obesity (Silver Spring) 2012; 20 : 1313-1318

[17] LaForgia J, Dollman J, Dale MJ et al. Validation of DXA body composition estimates in obese men and women. Obesity (Silver Spring) 2009; 17: 821-826

[18] Bosch TA, Burruss TP, Weir NL et al. Abdominal body composition difference in NFL football players. J Strength Cond Res 2014; 28 : 3313-3319

[19] Harriss DJ, Macsween A, Atkinson G. Standards for ethics in sport and exercise science research: 2020 update. Int J Sports Med 2019; 40: 813-817

[20] Toyo Medic Inc. Horizon QDR series of the product specification sheet by Hologic Inc. (Japanese version) Document No. AP15075000(178). 2015-01

[21] Cheung YM, Roff G, Grossmann M. Precision of the Hologic Horizon A dual energy $\mathrm{X}$-ray absorptiometry in the assessment of body composition. Obes Res Clin Pract 2020; 14: 514-518

[22] Faul F, Erdfelder E, Lang A-G et al. “G * Power 3: A flexible statistical power analysis program for the social, behavioral, and biomedical sciences. Behav Res Methods 2007; 39: 175-191

[23] Bland JM, Altman DG. Statistical methods for assessing agreement between two methods of clinical measurement. Lancet 1986; 1 : 307-310
[24] Tayler AE, Kuper H, Varma RD et al. Validation of dual energy X-ray absorptiometry measures of abdominal fat by comparison with magnetic resonance imaging in an Indian population. Plos One 2012; 7: e51042

[25] Goldberg EK, Fung EB. Precision of the Hologic DXA in the assessment of visceral adipose tissue. J Clin Densitom 2020; 23: 664-672

[26] Meredith-Jones K, Haszard J, Stanger N et al. Precision of DXA-derived visceral fat measurements in a large sample of adults of varying body size. Obesity (Silver Spring) 2018; 26: 505-512

[27] Knapp KM, Welsman JR, Hopkins SJ et al. Obesity increases precision errors in total body dual-energy $\mathrm{x}$-ray absorptiometry measurements. J Clin Densitom 2015; 18: 209-216

[28] Nana A, Slater G], Stewart AD et al. Methodology review: Using dual energy X-ray absorptiometry (DXA) for the assessment of body composition in athletes and active people. Int J Sport Nutr Exerc Metab 2014; 24: 198-215

[29] Steffes GD, Megura AE, Adams ] et al. Prevalence of metabolic syndrome risk factors in high school and NCAA Division I football players. J Strength Cond Res 2013; 27: 1749-1757

[30] Watanabe T, Morita A, Uno Y et al. Association of fatty liver and premetabolic syndrome in children [Japanese]. Japanese Journal of Medical Ultrasound Technology 2008; 33: 18-25

[31] Hurst RT, Burke RF, Wissner E et al. Incidence of subclinical atherosclerosis as a marker of cardiovascular risk in retired professional football players. Am J Cardiol 2010; 105: 1107-1111

[32] Kracht CL, Katzmarzyk PT, Staiano AE. Comparison of abdominal visceral adipose tissue measurements in adolescents between magnetic resonance imaging and dual-energy $X$-ray absorptiometry. Int J Obes (Lond) 2021; 45: 104-108

[33] Crabtree CD, Lafountain RA, Hyde PN et al. Quantification of human central adipose tissue depots: An anatomically matched comparison between DXA and MRI. Tomography 2019; 5: 358-366 